\title{
La sostenibilidad en el diseño de cadenas de suministro de biocombustibles
}

\author{
Lina María Tapia Barrera* \\ Jaime Acevedo Chedid ** \\ Hermes Araméndiz Tatis ${ }^{* * *}$ \\ Jaime Ararat Herrera***
}

Recibido: 10/04/2014 - Aceptado: 12/12/2014

\begin{abstract}
Resumen
El reto de utilizar fuentes de energía renovables implica que la producción de biocombustibles y el diseño de sus cadenas de suministro persigan beneficios ambientales y sociales, además de los beneficios netamente económicos. En el presente trabajo se revisaron algunas discusiones sobre los efectos de los biocombustibles en la sostenibilidad y trabajos de investigación que diseñaron cadenas de suministro de biocombustibles de primera y segunda generación mediante la optimización matemática, con el fin de analizar qué preocupaciones de tipo ambiental y social han sido consideradas en este problema. Al final se identificaron las técnicas de optimización más utilizadas y se encontró que la integración del concepto de sostenibilidad en el diseño de cadenas de suministro de biocombustibles a través de la modelación apenas comienza.
\end{abstract}

Palabras clave: sostenibilidad, diseño, cadena de suministro, biocombustibles, optimización.

1 Docente del Departamento de Ingeniería Industrial de la Universidad de Córdoba. Ingeniera Industrial, Especialista en Administración Total de la Calidad, Estudiante de MSc en Ingeniería de la Universidad Tecnológica de Bolívar-UTB. Montería. Correo: linatapia@correo.unicordoba.edu.co

** Docente del Departamento de Ingeniería Industrial de la Universidad Tecnológica de Bolívar. Ingeniero Industrial, Especialista en Mercadeo, MSc en Ingeniería Industrial, Doctorando en Ingeniería Industrial de la Universidad del Norte. Cartagena de Indias. Correo: jacevedo@unitecnologica.edu.co

*** Docente del Departamento de Ingeniería Agronómica de la Universidad de Córdoba. Ingeniero Agrónomo, MSc en Fitotecnia, Ph. D. Genética y Mejoramiento de Plantas. Montería. Correo: haramendis@correo.unicordoba. edu.co

**** Docente del Departamento de Ingeniería Industrial de la Universidad de Córdoba. Ingeniero Industrial, MBA del Externado de Colombia, Magíster en Ciencias de la Administración y Doctorando en Administración de la Universidad EAFIT. Correo: jararat@correo.unicordoba.edu.co 


\title{
Sustainability in the biofuels supply chains design
}

\begin{abstract}
The challenge of using renewable energy sources means that the production of biofuels and the design of their supply chains, should be pursue environmental and social benefits beyond the purely economic benefit. In this paper are revised some discussions about the impact of biofuels on sustainability and researching in the biofuel supply chains design of first and second generation via mathematical optimization, to analyze how environmental and social concerns have been considered in this problem. Finally, techniques most used optimization were identified and the review found that the concept of sustainability in the design of biofuel supply chains through modeling, is just beginning.
\end{abstract}

Keywords: sustainability, biofuels supply chain design, optimization. 


\section{INTRODUCCIÓN}

En 2010 el consumo mundial de energía fue de 524 cuatrillones de Btu, y se espera que aumente alrededor de $56 \%$ a 2040 ; la industria y el transporte son los principales responsables [1]. Entre tanto, las reservas de petróleo se agotan y el planeta avanza hacia un incremento devastador de $4{ }^{\circ} \mathrm{C}$ de la temperatura ambiente en menos de 50 años $[2,3,4]$, por lo que la humanidad enfrenta el reto de garantizar la seguridad energética y desacelerar el calentamiento global, ocasionados en cierta medida, por el uso intensivo de combustibles fósiles a lo largo del desarrollo industrial.

Así, los biocombustibles aparecen como una alternativa al problema de seguridad energética, con grandes beneficios ambientales debido a que se les atribuye la capacidad de aportar menos gases efecto invernadero que los combustibles derivados del petróleo. Sin embargo, su producción implica la expansión de cultivos (o frontera agrícola) y cambio en el uso de la tierra, lo cual puede derivar en algunos efectos indeseables para el ambiente y la sociedad, tales como deforestación, riesgos a la seguridad alimentaria, pérdida de biodiversidad, entre otros.

El presente artículo hace una revisión al estado del arte en el problema de diseño de cadenas de suministro de biocombustibles líquidos sostenibles, realizado con técnicas de optimización matemática. En primer lugar, se identificaron los trabajos de diseño de cadenas de suministro de bioetanol o biodiésel en bases de datos académicas; luego se revisó la modelación del problema y se seleccionaron aquellos trabajos que integraron preocupaciones económicas, ambientales y/o sociales en la formulación, con el fin de analizar cómo se ha desarrollado el concepto de sostenibilidad en este asunto.

Para alcanzar el objetivo, en la sección 1 se revisan algunas definiciones de los biocombustibles líquidos y en la sección 2 se discuten los principales efectos de su producción para el desarrollo sostenible. Luego, se describen las características principales de las cadenas de suministro de biocombustibles en la sección 3. En la sección 4, se revisa el alcance de los trabajos de diseño de estas cadenas cuando se utilizó la optimización matemática y se incluyeron intereses ambientales y/o sociales. Finalmente, se discuten los principales hallazgos de esta revisión y se identifican oportunidades de investigación en la administración de cadenas de suministro de biocombustibles o biofuel supply chain management.

\section{IMPORTANCIA DE LOS BIOCOMBUSTIBLES}

En su concepto más amplio, el término biocombustible se refiere a cualquier combustible de origen biológico que provenga de plantas o animales; sin embargo, suele restringirse el término para denominar a los biocarburantes líquidos (bioetanol y biodiésel) y al biogás [5]. 
El bio-alcohol (bioetanol) se produce principalmente de cultivos vegetales ricos en almidón y azúcares tales como maíz, caña de azúcar, yuca, trigo, papa, entre otros, los cuales se someten a una conversión enzimática que libera azúcares del almidón para su fermentación y posterior destilación [6]. El bio-aceite (biodiésel) se obtiene de especies oleaginosas como colza, palma de aceite, soya, girasol, jatropha y aceites de cocina usados; la tecnología de transformación más empleada es la transesterificación, aunque métodos como pirólisis, dilución con mezcla de hidrocarburos y microemulsión se encuentran en fase de experimentación con resultados positivos [7].

Los biocombustibles líquidos pueden ser de primera, segunda, tercera y cuarta generación según la biomasa utilizada para su obtención $[8,9,10]$. En la figura 1 se muestra esta clasificación.

Los biocombustibles de primera y segunda generación desarrollados hasta el momento garantizan seguridad energética, soporte agrícola e industrial, reducción de las importaciones de crudo, respuesta a la demanda de combustible y reducción de gases efecto invernadero (GEI); en cambio, estas condiciones no se pueden atribuir a los biocombustibles de tercera y cuarta generación debido a que se encuentran en fase de investigación, su producción es muy costosa, y no representan un escenario de inversión seguro $[10,11]$.

Los mayores productores de bioetanol son Estados Unidos y Brasil, en tanto que la Unión Europea, Argentina, Estados Unidos y Brasil son los cuatro principales productores de biodiésel. Se estima que la producción mundial de etanol se duplicará entre 2007 y 2017 alcanzando 125 billones de litros, y la del biodiésel aumentará de 11 a 24 billones de litros en el mismo periodo, en un escenario que no contempla cambios importantes en las políticas de incentivos a la producción y/o consumo de biocombustibles en Norteamérica y la Unión Europea [12,13].

El bioetanol y el biodiésel se utilizan mezclados o como sustitutos de la gasolina o el diésel para satisfacer necesidades en el transporte, principalmente. En el caso del bioetanol, la mezcla con gasolina comúnmente va del $5 \%$ al $10 \%$ (E5-E10), y se usa en vehículos con poca o ninguna modificación del motor, aunque los nuevos vehículos flexi-fuel pueden funcionar con mezclas hasta de $85 \%$; de forma similar, los porcentajes de volumen de biodiésel mezclado con petro-diésel varían desde $2 \%$ hasta $100 \%$ (B2, B100) y también pueden alimentar los vehículos diésel sin cambios en el motor [6]. Hoy, más de 50 países han adoptado objetivos o políticas de mezcla de biocombustibles y derivados del petróleo para regular su consumo, lo que ha significado cambios importantes en el suministro energético de algunas regiones; por ejemplo, Brasil logró que los biocombustibles abastecieran el $21 \%$ de su demanda interna de combustible 
para transporte terrestre durante 2008, mientras Estados Unidos y la Unión Europea cubrieron, respectivamente, el $4 \%$ y $3 \%$ de esta demanda en el mismo período [11].

En términos globales, el sector transporte es el segundo consumidor de energía después del sector industrial: representó el $26 \%$ de la energía total suministrada en 2010 y se estima que será el responsable de aproximadamente el $63 \%$ del aumento en la demanda de combustibles líquidos entre 2010 y 2040, lo cual está justificado por el comportamiento económico y poblacional de China e India [1].

Precisamente esta demanda de energía del sector transporte hace que los biocombustibles sean importantes para el desarrollo de sistemas de transporte sostenibles y de ahí su articulación a la solución de problemas globales, tales como el agotamiento del petróleo y el aumento de emisiones GEI. Sin embargo, su producción se identifica dentro de las causas del alza de precios en alimentos y uso inapropiado del suelo con aptitud agrícola que afectan la seguridad alimentaria, que es otra preocupación del desarrollo sostenible.

\section{LA SOSTENIBILIDAD Y LOS BIOCOMBUSTIBLES}

Desarrollo sostenible o sostenibilidad significa satisfacer las necesidades actuales sin comprometer la capacidad de las generaciones futuras de satisfacer las suyas, y en este sentido la Organización de Naciones Unidas (ONU) exhorta a todos los países a reducir el consumo de combustibles fósiles y a participar en compromisos que permitan sustituirlos por energías más limpias. Esta sostenibilidad generalmente se comprende en tres dimensiones: económica, ambiental y social $[14,15,16]$.

Las preocupaciones de sostenibilidad ambiental relacionadas con los biocombustibles son: emisiones GEI, uso y calidad de recursos hídricos, degradación del suelo y pérdida de la biodiversidad, principalmente $[10,17]$.

De acuerdo con la Agencia Internacional de Energía (IEA) [18], los biocombustibles son biodegradables en comparación con los derivados del petróleo, hacen combustión más limpia que la gasolina y disminuyen el material particulado lanzado al ambiente.

Algunas mediciones realizadas al ciclo de vida del etanol de maíz (cultivo, producción y consumo), demuestran que las emisiones GEI se reducen de $10 \%$ a $50 \%$ (si es de caña de azúcar esta reducción varía entre $70 \%$ y $120 \%$ y en el caso del biodiésel se espera una disminución de $30 \%$ a $70 \%$.

Sin embargo, se ha encontrado que la demanda de cultivos tradicionales como maíz y caña de azúcar destinados a biocombustibles aumenta al mismo tiempo los costos de almacenamiento de biomasa, la demanda de áreas de cultivo requeridas 
para el aprovisionamiento continuo de las bio-refinerías, el riesgo de cambio de uso del suelo y las emisiones GEI [19]. De hecho, llama la atención que el uso de la tierra aporte el $30 \%$ de las emisiones GEI globales, mientras que el sector transporte sea responsable del $13 \%[19,20]$.

Asimismo, se debe tener presente que la expansión de cultivos para obtención de biocombustibles puede afectar la diversidad genética que es la materia prima del mejoramiento genético, a su vez, necesario en la búsqueda de aumentar la productividad agrícola.

Ahora bien, si al incremento de la frontera agrícola y la huella de carbono por la expansión de los biocombustibles se suma la fuerte presión del aumento de la población mundial que demandará comida y bienes, entonces, es un imperativo que agricultura, producción de biocombustibles y desarrollo sostenible coincidan en el objetivo de usar controladamente la tierra para alimentos, energía y materiales.

En la sostenibilidad social, preocupa la divergencia entre la rentabilidad privada y los beneficios sociales que se obtendrían de la expansión de los biocombustibles $[21,22]$. Algunos trabajos advierten los efectos de la producción de biomasa sobre la seguridad alimentaria y la reducción de pobreza; otros estudian el vínculo entre los mercados de energía y la agricultura o analizan los cambios estratégicos en el uso y aptitud del suelo agrícola [23-29].

El Banco Mundial [30] y la Organización para la Alimentación y la Agricultura de Naciones Unidas (FAO) [31] atribuyen una responsabilidad importante a los biocombustibles en el aumento de los precios de los alimentos. Searchinger et al. [32] argumentan que en Estados Unidos el alza del precio del maíz incrementó la demanda de cultivos en otras partes del mundo, en especial de soya y trigo, debido a que se pasó de la industria de alimentación animal principalmente, a la producción de biocombustibles. Por su parte, Atabani et al. [7] afirman que la producción de biodiésel a partir de aceites comestibles, como de palma africana y soya, puede provocar un desequilibrio global en el suministro y demanda de alimentos. En contraste, Harvey \& Pilgrim [33] encuentran que la disminución de la productividad agrícola, la caída en las existencias de los cereales, el comercio especulativo y el establecimiento de barreras comerciales también pueden considerarse detonantes del alza en los precios de los alimentos.

Entre tanto, los biocombustibles se producen en zonas rurales donde hay oportunidades para la agricultura y siempre se presentan como alternativa de generación de empleo. Brasil demuestra que la industria de bioetanol soporta al menos $700 \mathrm{mil}$ puestos de trabajos directos y 200 mil indirectos, lo que representa 100 veces más empleo por unidad de energía que la industria del petróleo [34]. En Colombia, la 
agroindustria de palma de aceite genera 75 mil y la de caña de azúcar genera más de 100 mil entre directos e indirectos [35]. De esta manera, la relación entre producción de biocombustibles, precios de alimentos, disponibilidad de alimentos y uso del suelo resulta compleja, motiva diversas posiciones y continúa siendo materia de investigación.

Lo que sí es cierto, es que en el presente y en el futuro las relaciones económicas, sociales y ambientales de las apuestas productivas deben ser indivisibles, pues el desequilibrio en una dimensión significa grandes impactos en las otras. En este sentido, las tecnologías de producción y logística de los sistemas agrícolas destinados a alimentos, artículos no alimentarios y energía deben ser abordadas desde la seguridad alimentaria, la producción de energía verde, el desarrollo de productos sostenibles, la gestión de residuos y el reciclaje; de ahí que el enfoque de la cadena de suministro sea fundamental para integrar estos requerimientos en la producción agrícola, la transformación, la distribución y el consumo de los biocombustibles [36].

Por tanto, la investigación en gestión de cadenas de suministro sostenibles podría ayudar a resolver algunas de las preocupaciones ambientales y sociales relacionadas con la producción de biocombustibles.

\section{LAS CADENAS DE SUMINISTRO DE BIOCOMBUSTIBLES}

Las cadenas de suministro de biocombustibles están principalmente constituidas por granjas, centros de almacenamiento, plantas de bio-refinería, plantas de mezcla, puntos de venta y transportistas; y es común que estas unidades de negocio sean diferentes, tomen decisiones independientes y no siempre en función del logro de objetivos globales, lo que hace que este tipo de cadenas sean complejas, dados los conflictos de coordinación y cooperación entre sus eslabones [37].

El diseño de la cadena de suministro de biocombustibles incluye decisiones relacionadas con el tipo de biomasa a seleccionar, cuánto y de qué lugar tomarla, qué tecnología de producción instalar, dónde localizar las instalaciones y con qué capacidad, qué tipo y cantidad de biocombustible entregar y qué mercado satisfacer, con el fin de que la relación costo-beneficio de la producción de biocombustibles sea favorable [38]; como consecuencia, trabajos recientes relacionan los términos Biofuel Supply Chain (BSC) y Biofuel Supply Chain Management (BSCM) [10], [28], [39-41].

Gold \& Seuring [42] concluyen que los principales temas y desafíos relacionados con el diseño seguro y sostenible de la cadena de suministro de bioenergía están en las etapas de cosecha, recolección, almacenamiento, transporte y técnicas de pre-tratamiento, así como en el diseño de todo el sistema. Sin embargo, este enfoque sistémico no ha sido ampliamente adoptado en la literatura de planificación energética renovable, 
y las soluciones costo-beneficio para biocombustibles con un impacto mínimo en los alimentos y otros recursos naturales no han sido investigadas a fondo en la optimización aplicada al diseño de las cadenas de suministro [3], [43-44].

De acuerdo con Sharma et al. [45], se pueden identificar cuatro métodos de modelación aplicados en la gestión de la cadena de suministro: i) determinísticos, ii) estocásticos, iii) híbridos y iv) modelos dirigidos por Tecnologías de Información (IT-driven models). En los modelos determinísticos los parámetros son conocidos y se fijan con certeza. En los modelos estocásticos, también denominados probabilísticos, los parámetros son inciertos y aleatorios. Los modelos híbridos tienen elementos de los modelos deterministas y estocásticos. Estos incluyen los modelos teóricos de inventarios y modelos de simulación. Los IT-driven models integran y coordinan diversas fases de la planificación de la cadena de suministro en tiempo real, usando software de aplicación para tener la visibilidad de toda la cadena. Dentro de los ITdriven models, [46] destacan herramientas como Warehousing Management System (WMS), Transportation Management System (TMS), Enterprise Resource Planning (ERP), Geographic Information System (GIS), entre otras.

Actualmente, los modelos de optimización utilizados en la gestión de la cadena de suministro de biomasa se ocupan con mayor frecuencia de decisiones estratégicas, tales como ubicación, capacidad de plantas, y diseño de la red de suministro; en segundo lugar, estos modelos apoyan decisiones del nivel táctico y operativo. Las técnicas de modelación matemática y sus capacidades de solución han mejorado significativamente en los últimos años, y la visión integrada se está desarrollando [45].

Como el desarrollo sostenible y la aplicación de las técnicas de optimización en la gestión de cadenas de suministro actualizan la agenda de investigación de los biocombustibles, entonces, una revisión a los trabajos de diseño de BCS interesados en la sostenibilidad resulta útil para identificar líneas de atención e intervención en la materia.

\section{LA SOSTENIBILIDAD EN LOS MODELOS DE OPTIMIZACIÓN QUE APOYAN EL DISEÑO DE CADENAS DE SUMINISTRO DE BIOCOMBUSTIBLES: UN ESTADO DEL ARTE}

En la revisión de trabajos de diseño de BSC que han integrado preocupaciones de sostenibilidad ambiental y social, además de las económicas en su formulación, se encontró que en [39] elaboraron un modelo MILP para el diseño estratégico de la cadena de suministro de biocombustibles que persigue la minimización de los costos de funcionamiento de la cadena y del impacto ambiental en términos de emisiones GEI en el caso de etanol a base de maíz, tal como lo hicieron en [47] ocupándose de la minimización simultánea del costo total de una red de producción de azúcar/etanol y 
su desempeño ambiental durante todo el ciclo de vida del azúcar y el etanol, a través de un MILP.

En [28] buscaron la minimización de costos y la planificación estratégica de una cadena de suministro de bioetanol de segunda generación, desarrollando un MILP multi-periodo con variación de las demandas en el tiempo, que posibilita la mejor secuencia de apertura y expansión de bio-refinerías en un horizonte de tiempo dado; y similarmente, a través de un MILP, en [48] lograron planificar la producción de biocombustibles de segunda generación incluyendo incertidumbre y mejorando la formulación de una estructura de una red de suministro más compleja. Ambos trabajos integran la preocupación ambiental mediante el aprovechamiento de residuos para la obtención del biocombustible.

En contraste, en [43] desarrollaron un MILP multiobjetivo que permite planificar cadenas de suministro de etanol de segunda generación atendiendo a un objetivo por cada dimensión de la sostenibilidad. El objetivo económico fue la minimización del costo anualizado de operar la cadena, el ambiental buscó el menor nivel de emisiones GEI, y el social fue el aumento del número de puestos de trabajo locales. Seguidamente, [49] mediante un MILP buscaron el diseño óptimo de la cadena de suministro de etanol de segunda generación atendiendo a los mínimos costos y menores emisiones GEI. La frontera de Pareto óptima, resultante en ambos trabajos, revela que se puede llegar a la viabilidad económica de cadenas de suministro de bioetanol ambientalmente sostenibles que al tiempo son fuentes generadoras de empleo.

[41], [50] utilizaron modelos MILP para solucionar el problema de diseño de una cadena de suministro de biocombustible de primera y segunda generación en varios escenarios, con el objetivo de maximizar los beneficios económicos e incluyeron restricciones ambientales relacionadas con el uso del agua y tierra para la producción de biocombustibles (bioetanol o biodiesel) de primera y segunda generación.

Por su parte, en [39] utilizan también un MILP para solucionar el problema de diseño multi-período, multi-nivel y multi-objetivo de una cadena de suministro de bioetanol de primera y segunda generación que maximiza el beneficio económico y minimiza emisiones GEI en la producción del bioetanol. Después estos investigadores repiten con otro MILP para maximizar el valor presente neto del sistema, incorporando incertidumbre en el costo de la materia prima y el comercio de permisos de emisiones GEI. Sin embargo, restricciones u objetivos relacionados con el uso de suelos para la producción de alimentos no son tenidos en cuenta en sus trabajos [51].

En [44] modelaron el problema de localización de refinerías, teniendo en cuenta el comportamiento de juego cooperativo y no cooperativo de los agricultores. Los 
resultados demuestran que cuando las bio-refinerías y agricultores cooperan, se destina mayor porcentaje de la producción agrícola a generación de energía y favorece la economía de ambos grupos de interés.

Entre tanto, [52] incursiona en el problema de diseño de la cadena de suministro de biodiésel de primera generación con preocupación en la sostenibilidad, a partir de un modelo de programación no lineal (NLP) que incluye una restricción para el control de la degradación del suelo y rotación de cultivos. El estudio de caso realizado muestra que las asignaciones de tierras pueden mejorar significativamente la eficiencia de la cosecha y protegerla al mismo tiempo, aunque deja de lado el consumo de recurso hídrico, energía y huella de carbono del biocombustible.

Siguiendo la taxonomía de [45], en la tabla 1 se clasifican los trabajos anteriores según método de modelación, número de objetivos, aspecto de la sostenibilidad integrado en el diseño de la cadena de suministro y tipo de biocombustibles (sea etanol o diésel) de primera o segunda generación.

Tabla 1. Modelos de optimización aplicados en el problema de diseño de BSC que integraron preocupaciones de sostenibilidad ambiental y social

\begin{tabular}{|c|c|c|c|c|c|c|c|c|c|}
\hline \multirow[b]{2}{*}{ Autor y año } & \multirow[b]{2}{*}{$\begin{array}{l}\text { Método de } \\
\text { modelación }\end{array}$} & \multirow[b]{2}{*}{$\begin{array}{l}\text { Modelo según } \\
\text { número de } \\
\text { objetivos }\end{array}$} & \multicolumn{3}{|c|}{ Sustentabilidad } & \multicolumn{2}{|c|}{ Bioetanol } & \multicolumn{2}{|c|}{ Biodiesel } \\
\hline & & & 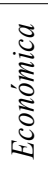 & 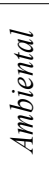 & $\begin{array}{l}\widetilde{\Xi} \\
\widetilde{\Xi} \\
\vdots\end{array}$ & $\underset{0}{5}$ & 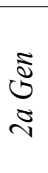 & $\underset{\Xi}{5}$ & 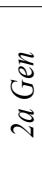 \\
\hline $\begin{array}{l}\text { Zamboni et al. } \\
(2009)\end{array}$ & MILP & Multi-objetivo & $\mathrm{x}$ & $\mathrm{x}$ & & $\mathrm{x}$ & & & \\
\hline Mele et al. (2009) & MILP & Multi-objetivo & $\mathrm{x}$ & $\mathrm{x}$ & & $\mathrm{x}$ & & & \\
\hline Huang et al. (2010) & MILP & Mono-objetivo & $\mathrm{x}$ & & & & $\mathrm{x}$ & & \\
\hline Kim et al. (2011) & MILP & Mono-objetivo & $\mathrm{x}$ & & & & $\mathrm{x}$ & & $\mathrm{x}$ \\
\hline You et al. (2011) & MILP & Multi-objetivo & $\mathrm{x}$ & $\mathrm{x}$ & $\mathrm{x}$ & & $\mathrm{x}$ & & \\
\hline $\begin{array}{l}\text { You \& Wang } \\
(2011)\end{array}$ & MILP & Multi-objetivo & $\mathrm{x}$ & $\mathrm{x}$ & & & $\mathrm{x}$ & & \\
\hline $\begin{array}{l}\text { Papapostolou et al. } \\
(2011)\end{array}$ & MILP & Mono-objetivo & $\mathrm{x}$ & $\mathrm{x}$ & & & & $\mathrm{x}$ & \\
\hline Akgul et al. (2012) & MILP & Mono-objetivo & $\mathrm{x}$ & $\mathrm{x}$ & & $\mathrm{x}$ & $\mathrm{x}$ & & \\
\hline $\begin{array}{l}\text { Giarola et al. } \\
(2011)\end{array}$ & Híbrido & Multi-objetivo & $\mathrm{x}$ & $\mathrm{x}$ & & $\mathrm{x}$ & $\mathrm{x}$ & & \\
\hline
\end{tabular}




\begin{tabular}{|c|c|c|c|c|c|c|c|c|c|}
\hline \multirow[b]{2}{*}{ Autor y año } & \multirow[b]{2}{*}{$\begin{array}{l}\text { Método de } \\
\text { modelación }\end{array}$} & \multirow[b]{2}{*}{$\begin{array}{l}\text { Modelo según } \\
\text { número de } \\
\text { objetivos }\end{array}$} & \multicolumn{3}{|c|}{ Sustentabilidad } & \multicolumn{2}{|c|}{ Bioetanol } & \multicolumn{2}{|c|}{ Biodiesel } \\
\hline & & & 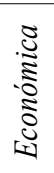 & 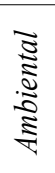 & $\begin{array}{l}\bar{\Xi} \\
\widetilde{\Xi} \\
\vdots \\
\leftarrow\end{array}$ & 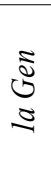 & 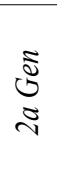 & 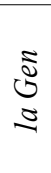 & $\begin{array}{l}\text { Iี } \\
\text { ป }\end{array}$ \\
\hline $\begin{array}{l}\text { Giarola et al. } \\
(2012)\end{array}$ & Híbrido & Multi-objetivo & $\mathrm{x}$ & $\mathrm{x}$ & & $\mathrm{x}$ & $\mathrm{x}$ & & \\
\hline Bai et al. (2012) & NLP & Mono-objetivo & $\mathrm{x}$ & & $\mathrm{x}$ & $\mathrm{x}$ & & & \\
\hline Avami (2012) & NLP & Mono-objetivo & $\mathrm{x}$ & $\mathrm{x}$ & & & & $\mathrm{x}$ & \\
\hline
\end{tabular}

Fuente: elaboración propia

Se puede notar que los modelos determinísticos tipo MILP, multiobjetivos y multiperíodos son los más utilizados, aunque los NLP ya empezaron a proponerse como alternativas en la modelación del problema de diseño BSC sostenibles.

Entre las preocupaciones ambientales se destaca el mercado de permisos de emisiones GEI, la reducción de emisiones de (kg de equiv/período de tiempo), la disponibilidad de recursos hídricos y suelos para evitar la degradación de los mismos como objetivos o restricciones en los modelos revisados. Aún falta explotar el aprovechamiento de residuos y la decisión de la generación de co-productos el fin de disminuir costos de operación y aumentar ingresos.

En la dimensión social, se integró la cooperación de agricultores como una variable endógena en la decisión de diseño de la cadena en un trabajo y la generación de empleo fue un objetivo en otro. Llama la atención que la restricción de uso de suelos se ha integrado en los modelos con un enfoque ambiental y no se relaciona con la problemática social de seguridad alimentaria, por lo que hace falta la inclusión de la aptitud y uso del suelo y la medición del impacto que tiene la producción de biocombustibles en la disponibilidad de alimentos, dentro del problema de diseño de BSC sostenibles.

De esta manera, las consideraciones sociales y la programación no lineal se convierten en las principales oportunidades de la investigación, y en futuras investigaciones deberían incluirse más especificidades de los sistemas agrícolas y biomasas, y otras consideraciones sociales, como desarrollo rural, inversión del Estado, reducción de pobreza y consideraciones de contratación de los agricultores con eslabones de la cadena aguas abajo (centros de acopio, industria y estaciones de consumo).

Aunque los biocombustibles de segunda generación posibilitan el aprovechamiento de residuos y esto contribuiría con la sostenibilidad ambiental, [28] y [48] no consideran restricciones o metas explícitas que cuantifiquen este impacto ambiental y/o social. 
Las validaciones o estudios de caso de los trabajos revisados son realizadas con sistemas productivos de bioetanol y biodiésel de primera generación en primer lugar, y del bioetanol de segunda generación, en segundo lugar. El comercio de biocombustibles de primera generación y la tradición de cultivos de biomasa en las regiones de los estudios de caso son las razones principales para que la parametrización y la validación de los modelos se lleven a cabo en este tipo de biocombustibles. Aunque se ha avanzado en la identificación de biomasas y desarrollo de tecnologías de producción de biodiésel de segunda generación, faltan trabajos que exploren el problema de diseño de la cadena de biocombustibles que, integrando tecnologías de biocombustibles de segunda generación, permitan la estimación de rendimientos económicos, ambientales y sociales.

Finalmente, todo trabajo que busque integrar cadenas de suministro de biocombustibles y sostenibilidad en un solo modelo o diseño enfrentará un gran desafío. No obstante, cualquier solución relacionada con la producción de biocombustibles deberá dirigirse en este sentido.

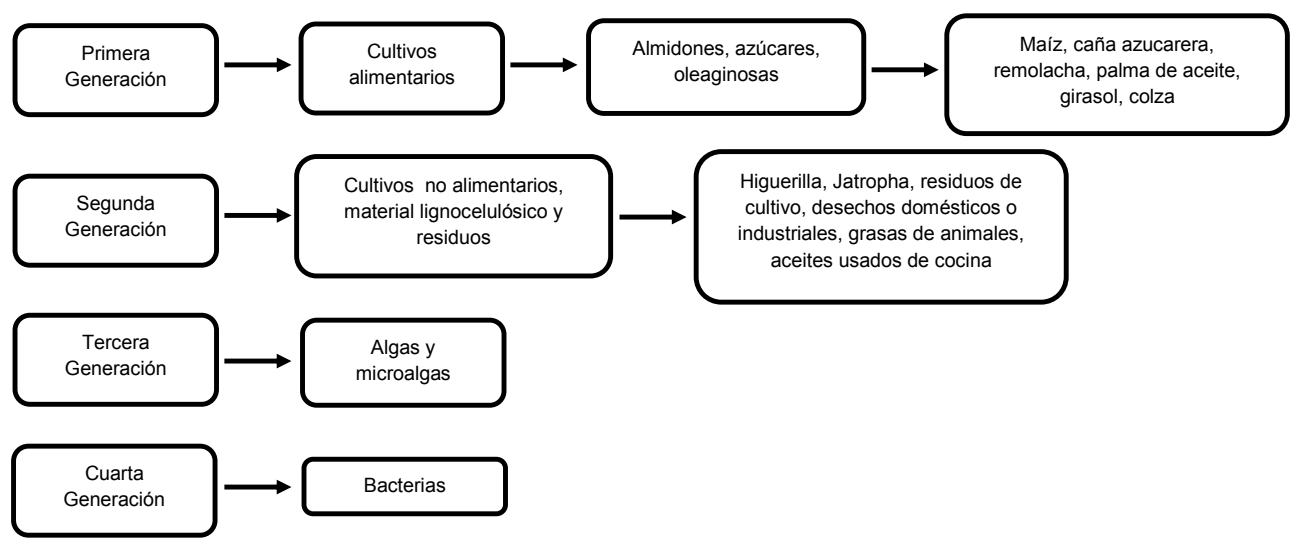

Figura 1. Clases de biocombustibles líquidos según la biomasa utilizada

Fuente: elaboración propia

\section{CONCLUSIONES}

La revisión permitió identificar que en la actualidad la decisión de invertir en la producción de biocombustibles se motiva por la búsqueda de seguridad energética y disminución de emisiones GEI principalmente, y la preocupación por el diseño de cadenas de suministro de biocombustibles que integre beneficios económicos, ambientales y sociales mediante la optimización matemática es reciente.

En el problema de diseño de BSC sostenibles, los modelos MILP multiobjetivos y multiperíodos han sido los métodos de modelación más utilizados, y los NLP apenas comienzan a proponerse. En estos trabajos las preocupaciones ambientales se han 
integrado con mayor frecuencia que los asuntos sociales, y las consecuencias de la producción de biocombustibles en seguridad alimentaria y reducción de los niveles de pobreza no se relacionan de manera directa en los modelos propuestos hasta ahora. Aún falta integrar consideraciones de las biomasas de segunda generación en este tipo de problema.

Debido a que el diseño de sistemas de producción de biocombustibles es determinante en el desarrollo de nuevos sistemas de transporte sostenibles, la optimización aplicada en el diseño de BSC actualiza la agenda de investigación de biocombustibles. Por tanto, los modelos de producción de biocombustibles con alto impacto ambiental y social representan un reto para investigadores, inversionistas y gobierno.

\section{REFERENCIAS BIBLIOGRÁFICAS}

[1] U.S. Energy Information Administration-EIA, “International Energy Outlook 2013”, 2013.

[2] A. Demirbas, "Biodiesel production from vegetable oils via catalytic and non-catalytic supercritical methanol transesterification methods", Prog. Energy Combust. Sci., vol. 31, N. ${ }^{\circ}$ 5-6, pp. 466-487, Jan. 2005.

[3] Organisation for Economic Co-operation and development (OECD) \& International Energy Agency (IEA), “World Energy Outlook 2006”, 2007.

[4] World Bank, "Turn Down the Heat: Why a $4{ }^{\circ} \mathrm{C}$ Warmer World Must be Avoided", 2012.

[5] I. promotion of the B. (PROBIO), "Estrategía para la promoción integrada de la cadena del biodiésel en la provincia de Huelva", 2008.

[6] I. E. A. (IEA), "Biofuel Production”, pp. 1-4, 2007.

[7] A. E. Atabani, A. S. Silitonga, I. A. Badruddin, T. M. I. Mahlia, H. H. Masjuki, and S. Mekhilef, "A comprehensive review on biodiesel as an alternative energy resource and its characteristics", Renew. Sustain. Energy Rev., vol. 16, N. 4, pp. 2070-2093, May 2012.

[8] A. Dermibas, Biohydrogen. 2009, p. 275.

[9] C. Álvarez, "Biocombustibles: desarrollo histórico-tecnológico, mercados actuales y comercio internacional", Econ. Inf., N..$^{\circ} 359$, pp. 63-89, 2009.

[10] I. Awudu and J. Zhang, "Uncertainties and sustainability concepts in biofuel supply chain management: A review”, Renew. Sustain. Energy Rev., vol. 16, N. 2, pp. 1359-1368, Feb. 2012.

[11] I. E. A. (IEA), “Technology Roadmap: Biofuels for Transport,” OECD Publishing, Jun. 2011.

[12] OECD-FAO agricultural outlook, 2008-2017, vol. 46, N. 07. 2009, pp. 46-3959-46-3959.

[13] Organisation for Economic Co-operation and development (OECD) \& Food and Agriculture Organization of the United Nation (FAO), "Agricultural Outlook", 2012. 
[14] United Nations, "Our common future", NGO Committee on Education of the Conference of NGOs from United Nations, p. 247, 1987.

[15] United Nations, "Protocolo de kyoto de la convención marco de las naciones unidas sobre el cambio climático", vol. 61702. p. 24, 1998.

[16] Comisión Europea, "Directiva 2009/28/CE del Parlamento Europeo sobre el Fomento del uso de energía procedente de fuentes renovables," vol. 2008, n. ${ }^{\circ}$ 2. Diario Oficial de la Unión Europea, pp. 16-62, 2009.

[17] S. Msangi, T. Sulser, M. Rosegrant, and R. Valmonte-Santos, "Global Scenarios for Biofuels: Impacts And Implications For Food Security And Water Use”, 2007.

[18] I. E. A. (IEA), “CO2 emissions from fuel combustion”, Paris, 2012.

[19] G. Tembo, F. M. Epplin, and R. L. Huhnke, "Integrative Investment Appraisal of a Lignocellulosic Biomass-to-Ethanol Industry”, vol. 28, N. ${ }^{\circ}$ 00, 2003.

[20] Intergovernmental Panel on Climate Change-IPCC, "Climate Change 2007-Mitigation of climate change", 2007.

[21] J. Janaun and N. Ellis, "Perspectives on biodiesel as a sustainable fuel”, Renew. Sustain. Energy Rev., vol. 14, N. ${ }^{\circ}$, pp. 1312-1320, 2010.

[22] A. Ervola, J. Lankoski, and M. Ollikainen, "Agriculture and climate change : Socially optimal production and land use", 2011.

[23] M. W. Rosegrant, S. Msangi, T. Sulser, and R. Valmonte-santos, "Biofuels and the Global Food Balance”, pp. 2005-2006, 2006.

[24] D. J. A. Johansson and C. Azar, "A scenario based analysis of land competition between food and bioenergy production in the US", 2007.

[25] R. Murphy, J. Woods, M. Black, and M. McManus, "Global developments in the competition for land from biofuels", Food Policy, vol. 36, pp. S52-S61, Jan. 2011.

[26] M. R. Dicks, J. Campiche, D. De La, T. Ugarte, C. Hellwinckel, H. L. Bryant, and J. W. Richardson, "Land Use Implications of Expanding Biofuel Demand", vol. 2, N. ${ }^{\circ}$ August, pp. 435-453, 2009.

[27] D. Rajagopal, S. Sexton, G. Hochman, and D. Roland-holst, "Model estimates food-versusbiofuel trade-off", vol. 5, N. ${ }^{\circ}$ December, pp. 199-201, 2009.

[28] Y. Huang, C. Chen, and Y. Fan, "Multistage optimization of the supply chains of biofuels", Transp. Res. Part E, vol. 46, N

$+{ }^{\circ} 6$, pp. $820-830,2010$.

[29] International Food Policy Research Institute-IFPRI, "Food Security, Farming, and Climate Change to 2050: Scenarios, Results, Policy Options", 2010.

Revista Ingenierías Universidad de Medellín 
[30] World Bank, “Commonwealth Finance Ministers Meeting”, London, 2008.

[31] Food and Agriculture Organization of the United Nation (FAO), "The state of food and agriculture", 2008.

[32] T. Searchinger, R. Heimlich, R. a Houghton, F. Dong, A. Elobeid, J. Fabiosa, S. Tokgoz, D. Hayes, and T.-H. Yu, "Use of U.S. croplands for biofuels increases greenhouse gases through emissions from land-use change", Science, vol. 319, N. ${ }^{\circ}$ 5867, pp. 1238-40, Feb. 2008.

[33] M. Harvey and S. Pilgrim, "The new competition for land : Food, energy, and climate change q", Food Policy, vol. 36, pp. S40-S51, 2011.

[34] E. F. De Almeida, J. V. Bomtempo, and C. M. D. S. E. Silva, "The Performance of Brazilian Biofuels: An Economic, Environmental", 2007.

[35] Fedebiocombustibles, “Avanza desarrollo de biocombustibles en el país”, 2013.

[36] J. E. G. Van Dam, B. De Klerk-engels, P. C. Struik, and R. Rabbinge, "Securing renewable resource supplies for changing market demands in a bio-based economy”, vol. 21, pp. 129-144, 2005 .

[37] K. Dautzenberg and J. Hanf, "Biofuel chain development in Germany: Organisation , opportunities, and challenges”, vol. 36, pp. 485-489, 2008.

[38] E. Iakovou, A. Karagiannidis, D. Vlachos, A. Toka, and A. Malamakis, "Waste biomass-toenergy supply chain management : A critical synthesis", Waste Manag., vol. 30, N. ${ }^{\circ}$ 10, pp. 1860-1870, 2010.

[39] S. Giarola, A. Zamboni, and F. Bezzo, "Spatially explicit multi-objective optimisation for design and planning of hybrid first and second generation biorefineries", Comput. Chem. Eng., vol. 35, N. ${ }^{\circ}$, pp. 1782-1797, Sep. 2011.

[40] H. An, W. E. Wilhelm, and S. W. Searcy, "Biofuel and petroleum-based fuel supply chain research : A literature review”, Biomass and Bioenergy, vol. 35, N. ${ }^{\circ}$ 9, pp. 3763-3774, 2011.

[41] O. Akgul, N. Shah, and L. G. Papageorgiou, "Economic optimisation of a UK advanced biofuel supply chain”, Biomass and Bioenergy, vol. 41, pp. 57-72, 2012.

[42] S. Gold and S. Seuring, "Supply chain and logistics issues of bio-energy production", J. Clean. Prod., vol. 19, n. ${ }^{\circ}$ 1, pp. 32-42, 2011.

[43] F. You, L. Tao, D. J. Graziano, and S. W. Snyder, “Optimal Design of Sustainable Cellulosic Biofuel Supply Chains : Multi-objective Optimization Coupled with Life Cycle Assessment and Input-Output Analysis", 2011.

[44] Y. Bai, Y. Ouyang, and J. Pang, "Biofuel supply chain design under competitive agricultural land use and feedstock market equilibrium”, Energy Econ., vol. 34, N. 5, pp. 1623-1633, 2012.

[45] B. Sharma, R. G. Ingalls, C. L. Jones, and a. Khanchi, "Biomass supply chain design and analysis: Basis, overview, modeling, challenges, and future", Renew. Sustain. Energy Rev., vol. 24, pp. 608-627, Aug. 2013. 
[46] H. Min and G. Zhou, "Supply chain modeling : past, present and future", vol. 43, pp. 231-249, 2002.

[47] R. Maria, D. B. Alves, C. Augusto, F. D. Mele, G. Guillén-gosálbez, and L. Jiménez, “Optimal Planning of the Sustainable Supply Chain for Sugar and Bioethanol Production”, pp. 597-602, 2009.

[48] J. Kim, M. J. Realff, and J. H. Lee, “Optimal design and global sensitivity analysis of biomass supply chain networks for biofuels under uncertainty", Comput. Chem. Eng., vol. 35, N. ${ }^{\circ}$, pp. 1738-1751, 2011.

[49] F. You and B. Wang, "Life Cycle Optimization of Biomass-to-Liquids Supply Chains with Distributed-Centralized Processing Networks”, pp. 1-63, 2011.

[50] C. Papapostolou, E. Kondili, and J. K. Kaldellis, "Development and implementation of an optimisation model for biofuels supply chain”, Energy, vol. 36, N. ${ }^{\circ} 10$, pp. 6019-6026, Oct. 2011.

[51] S. Giarola, N. Shah, and F. Bezzo, "Bioresource Technology A comprehensive approach to the design of ethanol supply chains including carbon trading effects", Bioresour. Technol., vol. 107, pp. 175-185, 2012.

[52] A. Avami, "A model for biodiesel supply chain: A case study in Iran", Renew. Sustain. Energy Rev., vol. 16, N. ${ }^{\circ}$ 6, pp. 4196-4203, 2012. 